\title{
Proteomic profile of human spermatozoa in healthy and asthenozoospermic individuals
}

\author{
Xiaodan Cao, Yun Cui, Xiaoxia Zhang, Jiangtao Lou, Jun Zhou, Huafeng Bei and Renxiong Wei* (D)
}

\begin{abstract}
Asthenozoospermia is considered as a common cause of male infertility and characterized by reduced sperm motility. However, the molecular mechanism that impairs sperm motility remains unknown in most cases. In the present review, we briefly reviewed the proteome of spermatozoa and seminal plasma in asthenozoospermia and considered post-translational modifications in spermatozoa of asthenozoospermia. The reduction of sperm motility in asthenozoospermic patients had been attributed to factors, for instance, energy metabolism dysfunction or structural defects in the sperm-tail protein components and the differential proteins potentially involved in sperm motility such as COX6B, ODF, TUBB2B were described. Comparative proteomic analysis open a window to discover the potential pathogenic mechanisms of asthenozoospermia and the biomarkers with clinical significance.
\end{abstract}

Keywords: Proteome, Spermatozoa, Sperm motility, Asthenozoospermia, Infertility

\section{Background}

Infertility is defined as the lack of ability to achieve a clinical pregnancy after one year or more of unprotected and well-timed intercourse with the same partner [1]. It is estimated that around $15 \%$ of couples of reproductive age present with infertility, and about half of the infertility is associated with male partner [2,3]. With the continuing world-wide increase in male infertility, it has become a major health problem attracting more clinical attention. Asthenozoospermia(AS) is a common cause of human male infertility characterized by reduced sperm motility with sperm motility $<50 \%$ or progressive motility $<25 \%$ [4]. The proteins involved in the normal physiology of the sperm motility is rather scarce and the molecular basis of asthenozoospermia is not yet fully understood [5-7]. The causes of poor sperm motility include abnormal metabolism in the testicular tissue or epididymis, structural deficiency in the sperm tail, and functional deficiency of the epididymis or other accessory sex glands [8-10]. Routine semen analysis on sperm motility is only a clinical indicator of male fertility and does not account for the underlying cause of defects associated with sperm movement. It is possible that individual protein defects in spermatozoa might cause

\footnotetext{
* Correspondence: cxd0319@163.com

Department of Clinical Laboratory, Ningbo Municipal Hospital of Traditional Chinese Medicine, Ningbo 315000, China
}

fertilization failure [4] and it has become clear that identifying the precise proteins and the pathways involved in sperm motility is needed [5].

\section{Application of proteomic techniques in male infertility}

Proteomic approaches, such as two-dimensional (2D) polyacrylamide gel electrophoresis, mass spectrometry (MS), matrix-assisted laser desorption ionization time of flight (MALDI-TOF) and isobaric tags for relative and absolute quantitation (iTRAQ) could be useful in identifying a wide range of the proteins responsible for diagnosis of sperm dysfunctions and the regulatory mechanism of male fertility [11-17] (Fig. 1). A number of studies have utilized high-throughput techniques to study protein alterations in fertile versus infertile groups, for example, normal versus malformed, capacitated versus incapacitated and low versus high sperm motility [11, 18-20]. These techniques offers the comprehensive understanding of sperm proteins with their particular structure and also provides a new view to study different functional states of sperm $[21,22]$. For example, Chan et al. performed MADLITOF to address altered protein phosphorylation and 

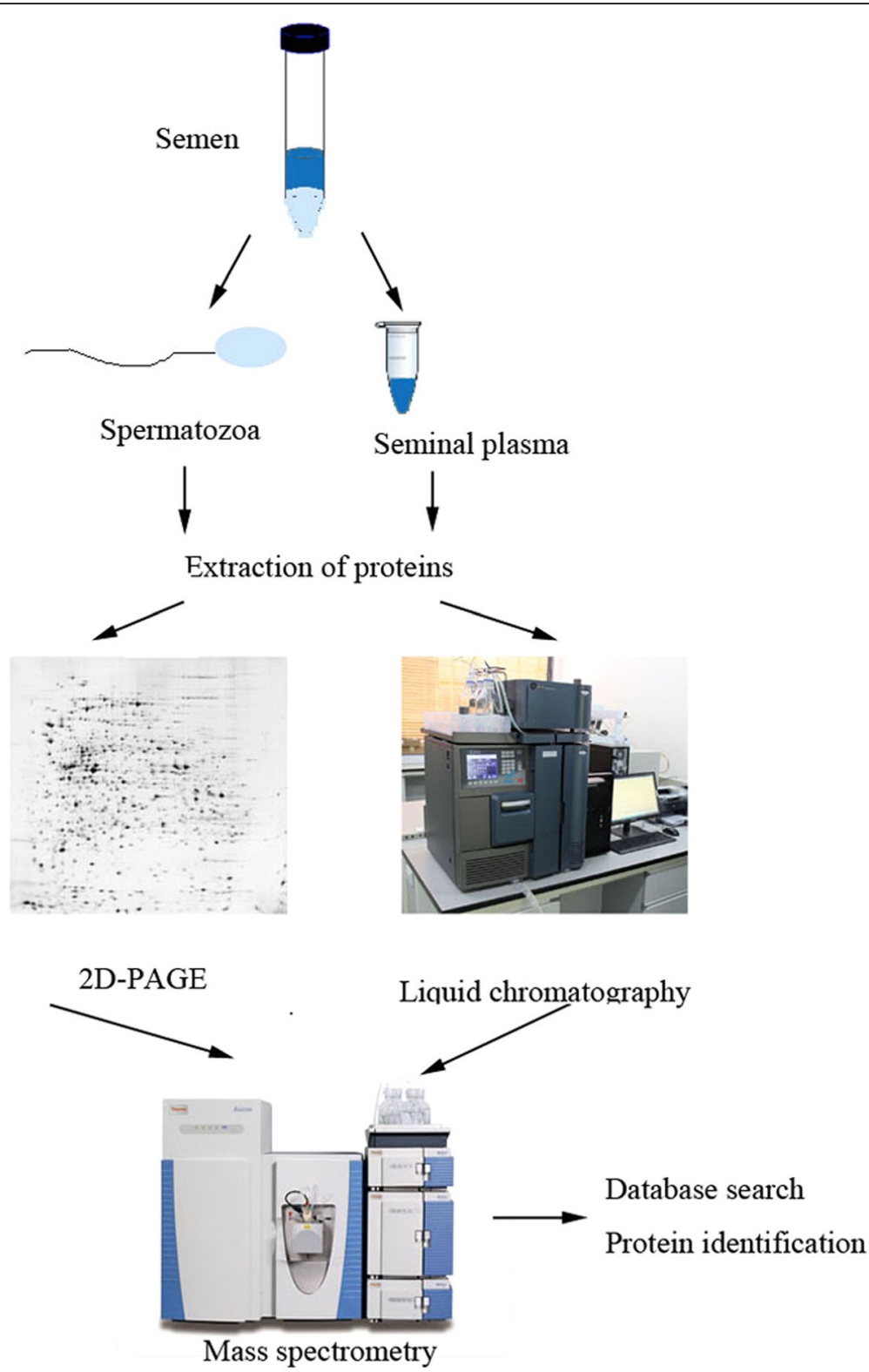

Fig. 1 Schematic diagram showing the proteomic analysis of the sperm cells and seminal plasma. After collection and liquefaction of ejaculated semen, the desired target components are purified through density gradients centrifugation. Proteins are extracted and separated by two-dimensional polyacrylamide gel electrophoresis (2D-PAGE) or liquid chromatography(LC), and then followed by identification using mass spectrometry and database search

aberrant sperm motility and 12 proteins were identified [18]. Secciani et al. employed MS/MS approach to analyze the protein profiles of capacitated versus ejaculated spermatozoa [23]. Sperm protein expression in asthenozoospermic patients and normozoospermic controls were compared, and several quantitative and qualitative significant variations were found [15]. Some of these differential proteins may be potential target proteins that could benefit further research on sperm function-related infertility and also shedding light on the key proteins involved in fertilization.

\section{Spermatozoal proteome in asthenozoospermia}

Sperm motility is an important prerequisite for successful fertilization. The proteomic studies on asthenozoospermic individuals are increasing and more and more proteins and pathways involved in sperm motility are identified (Table 1, Table 2). Hashemitabar et al. [24] investigated the protein expression profiles of human sperm tail from asthenozoospermic and normozoospermic individuals using the MALDI-TOF-TOF approach. Among the fourteen differentially expressed proteins, eleven proteins [(A-kinase anchor protein 4 (AKAP4), 
Table 1 Sperm proteins with a significantly higher or lower expression in asthenozoospermia in different proteomic studies

\begin{tabular}{|c|c|c|c|}
\hline \multirow[t]{2}{*}{ Study } & \multirow[t]{2}{*}{ Method } & \multicolumn{2}{|l|}{ Identified proteins } \\
\hline & & up-regulated in astheno & down-regulated in astheno \\
\hline Hashemitabar et al. & $\begin{array}{l}\text { 2D electrophoresis } \\
\text { MALDI-TOF-TOF analysis }\end{array}$ & CLU, KRT1, ASRGL1 & $\begin{array}{l}\text { AKAP4, ODF2, TUBB2B, COX6B, GSTMU3, } \\
\text { PHGPX, GAPD-S, VDAC2, HSPA2, HSPA9 } \\
\text { and SPANXB }\end{array}$ \\
\hline Siva et al. & 2D PAGE MALDI MS/MS analysis & TRIS, GPK2, SCOT1, unidentified & TUBB2C, tektin 1, PSMA3, HSPA2 \\
\hline Marti'nez-Heredia et al. & 2D electrophoresis MS analysis & $\begin{array}{l}\text { CLUpre, DLDpre, FHpre, HSPA2, } \\
\text { IMPA1, PSMB3, SEMG1pre, TEX12, } \\
\text { MPST/ECH1pre }\end{array}$ & $\begin{array}{l}\text { ACTB, ANXA5, COX6B, histone H2A, PIP, } \\
\text { PIPpre, calcium binding protein-S100A9 }\end{array}$ \\
\hline Shen et al. & $\begin{array}{l}\text { 2D electrophoresis } \\
\text { MALDI-TOF analysis }\end{array}$ & PIPpre, flagellin, GPX4, GAPDH & $\begin{array}{l}\text { TEKT4, Lacto, ODF2, SPANXB, PGK2, GRP78, } \\
\text { Hsp70, DJ-1, CAB2, Actin, Heat shock protein } \\
70 \text { testis variant }\end{array}$ \\
\hline Zhao et al. & 2D electrophoresis MALDI-TOF analysis & $\begin{array}{l}\text { PGM2, TPI, GOT-1, ODF, GS, } \\
\text { semenogelin I precursor }\end{array}$ & IDH-a, CA-II, GDI-1, MSS1 \\
\hline
\end{tabular}

outer dense fiber 2 (ODF2), tubulin beta 2B (TUBB2B), cytochrome c oxidase subunit 6B (COX6B), glutathione S-transferase $\mathrm{Mu} 3$ (GSTMu3), phospholipid hydroperoxide glutathione peroxidase (PHGPx), glyceraldehyde3-phosphate dehydrogenase, testis-specific (GAPD-S), voltage-dependent anion-selective channel protein 2 (VDAC2), heat shock-related $70 \mathrm{kDa}$ protein 2 (HSPA2), stress-70 protein, mitochondrial (HSPA9), Sperm protein associated with the nucleus on the $\mathrm{X}$ chromosome $\mathrm{B}$ (SPANXB)] had increased amounts in normal controls, and three proteins [clusterin (CLU), keratin, type II cytoskeletal 1 (KRT1), isoaspartyl peptidase/L-asparaginase (ASRGL1)] had higher expression levels in asthenozoospermic samples. In the functional categorization, the majority fall into five groups: energy and metabolism (COX6B, GAPDS, PHGPx), movement and structural organization (TUBB2B, ODF2, AKAP4, KRT1, CLU), stress response and turn over (HSPA2, HSPA9), signaling and transport (VDAC2), and antioxidant activity (GSTMu3).

Proteins responsible for energy and metabolism (COX6B, GAPDS and PHGPx), associated with the production of ATP in distal regions of the flagellum $[25,26]$, had a decreased expression in asthenozoospermic sperm samples. The decreased level might affect the structure of fibrous sheath and ATP production, which might impair sperm motility. VDAC family proteins with an ATP binding site regulates mitochondrial outer membrane permeabilization to ions and ATP molecules [27, 28] and knockout of the VDAC2 and VDAC3 gene resulted in significantly reduced sperm motility and embryo development[29], suggesting that

Table 2 Summary of Gene Ontology $(\mathrm{GO})$ analysis of differential sperm proteins in asthenozoospermia in different proteomic studies

\begin{tabular}{lll}
\hline Study & GO term & Protein names \\
\hline Hashemitabar et al. & energy and metabolism & COX6B, GAPDS, PHGPX \\
& movement and structural organization & TUBB2B, ODF2, AKAP4, KRT1, CLU \\
& stress response and turn over & HSPA2, HSPA9 \\
& signaling and transport & VDAC2 \\
& antioxidant activity & GSTMu3 \\
Siva et al. & energy and metabolism & TRIS, GPK2, SCOT1, unidentified \\
& movement and organization & TUBB2C, tektin 1 \\
Marti'nez-Heredia et al. & energy production & PSMA3, HSPA2 \\
& structure and movement & COX6B, DLDpre, Fhpre, ECH1 pre \\
Zhao et al. & cell signalling and regulation & ACTB, H2A, PIP, PIPpre, SEMG1 \\
& metabolic enzymes & ANXA5, S100A9, IMPA1 \\
& structure & IDH-a, PGM2, TPI, GOT-1, CA-II \\
& other & GDI-1,ODF \\
\hline
\end{tabular}


VDACs are indispensable for sperm energy metabolism and successful fertilization.

Sperm is a highly polarized cell and its motility is fully dependent on flagellum. The ability of sperm to move forward is crucial for the successful fertilization of an egg [30]. The reduction of sperm motility in asthenozoospermic men has been attributed to factors such as structural defects in the sperm-tail protein components [8]. Sperm flagellum consists of an axoneme surrounded in distinct regions by accessory structures such as the fibrous sheath and ODF. AKAP3 and AKAP4 are the most abundant structural proteins in the fibrous sheath and have anchoring sites for cyclic-AMP-(cAMP)dependent protein kinase that increases tyrosine phosphorylation of sperm proteins and regulating flagellar function [31]. Deleting AKAP4 gene in male mice caused dysplasia of the fibrous sheath (DFS) which lead to defective sperm motility [32]. Absence of AKAP4 resulted in loss of effective sperm motility because cAMPdependent signaling processes fail to be associated with the fibrous sheath [33]. The fibrous sheath is involved in regulating sperm maturation, motility and capacitation and AKAP4 could influence the sperm locomotion and might be a biomarker of understanding prefertilization events. Numerous polypeptides such as ODF1 and ODF2 constitute the ODF sheath which help to preserve the elastic rigidity of sperm flagellum [34, 35]. An ultra structural study of asthenozoospermia individuals with DFS showed the abnormal extension of ODF to the principal piece[36]. Reduction of one or more ODF proteins might result in decreased elasticity of sperm flagellum and cause nonfunctional tails [37]. There is a possibility for a screening regarding the distribution of sperm tail proteins related to motility disorder.

Tubulin is known to play a key role in the formation of microtubules of flagellum. TUBB2C is a major cytoskeletal protein present in the head and flagellum of spermatozoa and existed actual influences on sperm motility $[38,39]$. TUBB2B, expressed in post-mitotic cells in male germ cells, are essential for the the formation of meiotic spindles and cytoplasmic microtubules and also share in the organization of $9+2$ of the axonemal microtubule in sperm tail [40]. Sperm in asthenozoospermic patients were defective in motility might due to the lower expression of tubulin with structural defects in sperm flagellum like dysplasia of the fibrous sheath (DFS) with missing axonemal central pairs and complete distortion of $9+2$ axonemal structure [36].

HSP family proteins are stress response proteins which protect the cell against oxidative stress and pathological changes [41]. HSPA9 is predominant in the mitochondrial matrix and required during protein translocation into mitochondria and the refolding of mitochondria proteins [42]. GSTMu3 is a kind of antioxidant proteins and participates in the detoxification of toxins, drugs and oxidative stress products [43]. The down-regulated of HSPA9 and GSTMu3 in asthenozoospermia could lead to the accumulation of oxidative stress products and impair sperm motility.

Siva et al. [44] employed 2D PAGE MALDI MS/MS analysis to compare the sperm proteome of asthenozoospermic and normozoospermic patients. Eight proteins showing changes in levels between two groups were revealed and the differential proteins belonged to three functional groups: energy and metabolism [triose-phosphate isomerase(TRIS), glycerol kinase, testis specific 2 (GPK2) and succinyl-CoA:3-ketoacid CoA transferase 1 (SCOT1), unidentified], movement and organization [tubulin beta-2C chain(TUBB2C) and tektin 1] and protein turnover, folding and stress response [proteasome subunit alpha type 3 (PSMA3) and HSPA2]. It was interesting to note that proteins falling in the 'energy and metabolism' group are higher in asthenozoospermic individuals and the high expression may be because of the redundant cytoplasm (droplets) contained in immaturation spermatozoa, while proteins with altered intensity in other two functional groups are higher in normozoospermic donors. TUBB2C, TPIS, HSPA2 and PSMA3 have been reported to get S-nitrosylated in human sperm during capacitation [17], indicating an involvement of these proteins in various sperm functions associated with capacitation, including sperm motility. HSPA2 has been shown to be a marker for sperm maturity [45] with lower amounts in oligoteratozoospermic men [46].

Proteasomes have been detected in sperm from various species and the major function of proteasome is to degrade nuclear and cytosolic ubiquitinated proteins [47-49]. Its low expression in asthenozoospermia might lead to the accumulation of higher ubiquitinated molecules and was related to sperm motility [48]. Studies on the sperm proteome from asthenozoospermic samples have revealed that the relative expression of some component of the proteasome complex is different. Zhao et al. [15] reported that the $26 \mathrm{~S}$ protease regulatory subunit 7 (MSS1) is decreased in asthenozoospermic patient, in contrast to the study by Marti'nez-Heredia et al. [50] which showed that PSMB3 is increased in asthenozoospermia. The different trend for proteasome subunit is not clear and may be attributed to ethnic differences (studies by Siva and Zhao group worked on Asian population and Martı'nez-Heredia et al., worked on European population). Further study on a larger sample size would be required to confirm these findings.

Martı'nez-Heredia et al. [50] compared the expression of asthenozoospermic samples to that of semen donor controls using two-dimensional proteomic analysis and seventeen protein spots have been identified at different amounts. Of these proteins identified, 
COX6B, dihydrolipoamide dehydrogenase precursor (DLDpre), fumarate hydratase precursor (FHpre) and 3mercapto-pyruvate sulfurtransferase/dienoyl-CoA isomerase precursor (MPST/ECH1pre) constitute the 'energy production' group; cytoskeletal actin-B (ACTB), histone $\mathrm{H} 2 \mathrm{~A}$, prolactin-inducible protein (PIP), prolactin-inducible protein precursor (PIPpre), and semenogelin 1 precursor (SEMG1pre) constitute 'structure and movement' group; and the 'cell signalling and regulation' group is constituted by annexin-A5 (ANXA5), calcium binding proteinS100A9, and inositol-1 monophosphatase (IMPA1). It is interesting to note that six of these identified proteins are precursor forms [(DLDpre, FHpre, ECH1pre, PIPpre, SEMG1pre and clusterin precursor (CLUpre)] of mature proteins, which could suggest the presence of a generalized post-translation processing problem of these proteins. The accumulation of precursors in asthenozoospermic patients also might lead to the deregulation of some downstream proteins. DLDpre and Fhpre, associated with the major sources of ATP production in sperm, were upregulated in asthenozoospermic patients, which could be involved with a potential decrease in the mature protein and the failure of tail movement of sperm in asthenozoospermia [51, 52]. The results together with those of Zhao et al. [15] demonstrate the existence of a general deregulation in the pathways involved in energy and metabolism in asthenozoospermic patients.

PIP is a $17-\mathrm{kDa}$ glycoprotein present in human seminal plasma. Its aspartyl-proteinase nature suggests its specificity to fibronectin that is one of the major protein constituents of the seminal coagulum [53-55], which indicates that PIP could contribute to the specifical degradation of fibronectin during liquefaction. Lower expression of PIP in asthenozoospermia might result in an incomplete liquefaction of the ejaculate and make a restriction for the movement of spermatozoa.

2D electrophoresis MALDI-TOF analysis was performed to compare sperm protein composition in asthenozoospermic patients with that of normozoospermic samples $[15,56]$. Sixteen differentially expressed protein were identified by Shen et al. and tektin 4 (TEKT4) and ODF2 appeared only in normozoospermic samples [56]. Tektins and ODFs form the main components of the cytoskeleton in sperm flagella, and the reduction of sperm motility in asthenozoospermic men has been attributed to factors such as abnormal expression of the structural constituents of cytoskeleton [8]. $78 \mathrm{kDa}$ glucose-regulated protein (GRP78), belonging to HSP protein located on endoplasmic reticulum(ER), is down-regulated in asthenozoospermic samples. GRP78 was able to bind to the acrosomal region of capacitated human spermatozoa and to modulate the binding interaction between sperm and the zona pellucida, which might affect intracellular signaling transduction and the capacitation of sperm $[57,58]$.
Ten differentially expressed proteins involved in the regulation of sperm motility were identified by Zhao et al. and these proteins were distributed into three categories: metabolic enzymes, tructure-associated proteins and other functional proteins [15]. Contrary to the previous studies, ODF protein showed higher expression in asthenozoospermia in this study and the disturbance of ODF protein may be associated with sperm motility defect. Half of the identified proteins are related with ATP metabolism to support sperm movement. The overexpression of phosphoglycerate mutase 2 (PGM2), triosephosphate isomerase(TPI) and glutamate oxaloacetate transaminase-1(GOT-1) in asthenozoospermic patients may be a compensatory reaction to the reduced motility sperm and weakened TCA cycle associated with low isocitrate dehydrogenase subunit $\alpha$ (IDH- $\alpha)$ expression [15].

\section{Post-translational modifications in spermatozoa of asthenozoospermia}

Post-translational modifications especially phosphorylation play an important role in sperm motility and protein phosphorylation is reported to be a prerequisite for spermatozoa to fertilize an egg. Chan et al. [23] performed 2D electrophoresis MALDI-TOF MS approach to analyze motility and protein phosphorylation in healthy and lowmotility-sperm and identified twelve spots as having differential phosphorylation. Among these 12 proteins, 10 proteins were hypophosphorylated in asthenozoospermic sperm, while 2 proteins exhibited a relatively lower phosphorylation level in normal sperm. Parte et al. [59] investigated the expression of phosphoproteins in normal- and asthenozoo-sperm by Nano UPLC-MSE and Ingenuity Pathways Analysis to identify the differential phosphoproteins and the key pathways regulating sperm motility. 66 phosphoproteins were significantly different of which 27 proteins were decreased and 39 proteins were increased in asthenozoosperm compared with those in normal samples. The differentially regulated proteins contained the family of HSPs, cytoskeletal proteins and proteins involved in the fibrous sheath and energy metabolism. Pathway analysis indicated that carbohydrate and energy metabolism, cyclic AMP signaling, PI3K/AKT signaling and pathway regulating actin based motility by Rho were significantly altered. The data identified demonstrated that the signature molecules of metabolic enzymes, fibrous sheath associated proteins and cytoskeletal proteins have the potential as biomarkers for diagnosing axonemal defects, a defective fibrous sheath, and abnormal mitochondria in asthenozoospermia.

\section{Proteome in the seminal plasma of asthenozoospermia}

Seminal plasma is a mixture of secretions from the testis, epididymis and several male accessory glands and 
a growing number of seminal plasma proteins have been shown to be related with sperm motility. To further explore the impact of post-testicular processes on sperm motility, seminal plasma proteins from asthenozoospermia individuals and healthy men were collected and analyzed by LC-MS/MS. 101 differentially expressed proteins were identifed and Chain A, Human Dj-1 with sulfinic acid (DJ-1) was the most downregulated protein in the seminal plasma of asthenozoospermic patients. Most of these proteins enriched in the hydrolase activity, catalytic activity and enzyme regulator activity. In addition, the levels of reactive oxygen species (ROS) were higher in asthenozoospermia men and these data suggest that downregulation of the DJ-1 protein could lead to increased oxidative stress for spermatozoa which would influence the quality of the semen and sperm motility [60]. Saraswat et al. [61] have performed a differential proteomic analysis of the sperm and seminal plasma in normozoospermia and asthenozoospermia samples and identified 667 proteins for label-free analysis in sperm and 429 proteins in seminal plasma. Statistical and mathematical analysis including principal component analysis, OPLS-DA (S-Plot), ROC Curve analysis and self-organizing maps analysis were performed on the dataset. Various pathways were enriched in the proteomic datasets and some of these pathways were gluconeogenesis, glycolysis, stress response, nucleosome assembly, axoneme activation and focal adhesion assembly. The studies elucidated the pathways underlying the sperm motility which can lead to identification of novel treatment avenues.

\section{Conclusion}

In this review, we have compared data of proteome studies from asthenozoospermic men and normal individuals that evaluated the protein profile that controls infertility. Studies compiled in this work would contribute appreciably to the presently limited information available about molecular mechanism underlying sperm motility. There is a need to extend these studies to get a consistent view on the specific proteins involved in asthenozoospermia and their characterization could merit further investigation on sperm motility-related infertility. Ultimately, these findings will contribute towards the development of novel diagnostic markers of male infertility.

\footnotetext{
Abbreviations

AKAP4: A-kinase anchor protein 4; ANXA5: annexin-A5; ASRGL1: Isoaspartyl peptidase/L-asparaginase; CAB2: XPA binding protein 2, isoform CRA_b; CAII: Carbonic anhydrase II; CLU: Clusterin; CLUpre: Clusterin precursor; COX6B: Cytochrome c oxidase subunit 6B; DFS: Dysplasia of the fibrous sheath; DJ-1: Chain A, Human Dj-1 with sulfinic acid; DLDpre: Dihydrolipoamide dehydrogenase precursor; Fhpre: Fumarate hydratase precursor; GAPDH: Glyceraldehyde-3-phosphate dehydrogenase; GAPD-S: Glyceraldehyde-3-phosphate dehydrogenase, testis-specific; GDI1: Rho GDP-dissociation inhibitor 1; GKP2: Glycerol kinase, testis specific 2; GOT-1: Glutamate oxaloacetate transaminase-1; GPX4: Phospholipid
}

hydroperoxide glutathione peroxidase; GRP78: 78 kDa glucose-regulated protein; GS: Glutamine synthetase; GSTMu3: Glutathione S-transferase Mu 3; HSP: Heat shock protein; HSPA2: Heat shock-related 70 kDa protein 2; HSPA9: Stress-70 protein, mitochondrial; IDH-a: Isocitrate dehydrogenase subunita; IMPA1: Inositol-1 monophosphatase; iTRAQ: Isobaric tags for relative and absolute quantitation; KRT1: Keratin, type II cytoskeletal 1; Lacto: Lactoferrin; MALDI-TOF: Matrix-assisted laser desorption ionization time of flight; MPST/ECH1 pre: 3-mercapto-pyruvate sulfurtransferase/dienoyl-CoA isomerase precursor; MS: Mass spectrometry; MSS1: 26S protease regulatory subunit 7; ODF: Outer dense fiber; PGK2: Phosphoglycerate kinase 2; PGM2: Phosphoglycerate mutase 2; PIP: Prolactin-inducible protein; PIPpre: Prolactin-inducible protein precursor; PSMA: Proteasome subunit alpha; PSMB3: Proteosome subunit beta 3; ROS: Reactive oxygen species; SCOT: Succinyl-COA:3-ketoacid COA transferase; SEMG1 pre: Semenogelin 1 precursor; SPANXB: Sperm protein associated with the nucleus on the $X$ chromosome B; TEKT4: Tektin 4; TEX12: Testis expressed sequence 12; TPI: Triosephosphate isomerase; TUBB2B: Tubulin beta 2B; TUBB2C: Tubulin beta 2C; VDAC2: Voltage-dependent anion-selective channel protein 2

\section{Acknowledgments}

Not applicable

\section{Funding}

This work was supported by the Natural Science Foundation of Ningbo (No. 2016A610200).

\section{Availability of data and materials}

All data generated or analyzed during this study are included in this published article.

\section{Authors' contributions}

XDC, XXZ, JTL, JZ and HFB were involved in data acquisition, analysis and manuscript drifting. XDC was the major contributor in writing the manuscript. YC and RXW approved the final manuscript. All authors read and approved the final manuscript.

Ethics approval and consent to participate

Not applicable

\section{Consent for publication}

Not applicable

\section{Competing interests}

The authors declare that they have no competing interests.

\section{Publisher's Note}

Springer Nature remains neutral with regard to jurisdictional claims in published maps and institutional affiliations.

Received: 28 November 2017 Accepted: 22 February 2018

Published online: 27 February 2018

\section{References}

1. Brugh VM 3rd, Lipshultz LI. Male factor infertility: evaluation and management. Med Clin North Am. 2004;88(2):367-85.

2. Luk BH, Loke AY. A review of supportive interventions targeting individuals or couples undergoing infertility treatment: directions for the development of interventions. J Sex Marital Ther. 2016;42(6):515-33.

3. Esteves SC, Chan P. A systematic review of recent clinical practice guidelines and best practice statements for the evaluation of the infertile male. Int Urol Nephrol. 2015:47(9):1441-56.

4. World Health Organization. Laboratory manual of the WHO for the examination of human semen and sperm-cervical mucus interaction. Ann Ist Super Sanita. 2001; 37(1):I-XII, 1-123.

5. Luconi M, Forti G, Baldi E. Pathophysiology of sperm motility. Front Biosci. 2006:11:1433-47.

6. Baccetti B, Collodel G, Estenoz M, Manca D, Moretti E, Piomboni P. Gene deletions in an infertile man with sperm fibrous sheath dysplasia. Hum Reprod. 2005;20(10):2790-4.

7. Rousseaux S, Caron C, Govin J, Lestrat C, Faure AK, Khochbin S. Establishment of male-specific epigenetic information. Gene. 2005;345(2):139-53. 
8. Ryder TA, Mobberley MA, Hughes L, Hendry WF. A survey of the ultrastructural defects associated with absent or impaired human sperm motility. Fertil Steril. 1990;53(3):556-60.

9. Comhaire FH, Mahmoud AM, Depuydt CE, Zalata AA, Christophe AB. Mechanisms and effects of male genital tract infection on sperm quality and fertilizing potential: the andrologist's viewpoint. Hum Reprod Update. 1999;5(5):393-8.

10. Infante JP, Huszagh VA. Synthesis of highly unsaturated phosphatidylcholines in the development of sperm motility: a role for epididymal glycerol-3phosphorylcholine. Mol Cell Biochem. 1985;69(1):3-6.

11. Pixton KL, Deeks ED, Flesch FM, Moseley FL, Bjorndahl L, Ashton PR, et al. Sperm proteome mapping of a patient who experienced failed fertilization at IVF reveals altered expression of at least 20 proteins compared with fertile donors: case report. Hum Reprod. 2004;19(6):1438-47.

12. Baker MA, Witherdin R, Hetherington L, Cunningham-Smith K, Aitken RJ. Identification of post-translational modifications that occur during sperm maturation using difference in two-dimensional gel electrophoresis. Proteomics. 2005;5(4):1003-12.

13. Johnston DS, Wooters J, Kopf GS, Qiu Y, Roberts KP. Analysis of the human sperm proteome. Ann N Y Acad Sci. 2005;1061:190-202.

14. Capkova J, Elzeinova F, Novak P. Increased expression of secretory actinbinding protein on human spermatozoa is associated with poor semen quality. Hum Reprod. 2007;22(5):1396-404.

15. Zhao C, Huo R, Wang FQ, Lin M, Zhou ZM, Sha JH. Identification of several proteins involved in regulation of sperm motility by proteomic analysis. Fertil Steril. 2007;87(2):436-8.

16. Baker MA, Reeves G, Hetherington L, Muller J, Baur I, Aitken RJ. Identification of gene products present in triton X-100 soluble and insoluble fractions of human spermatozoa lysates using LC-MS/MS analysis. Proteomics Clin Appl. 2007;1(5):524-32.

17. Lefievre L, Chen Y, Conner SJ, Scott JL, Publicover SJ, Ford WC, et al. Human spermatozoa contain multiple targets for protein S-nitrosylation: an alternative mechanism of the modulation of sperm function by nitric oxide? Proteomics. 2007;7(17):3066-84

18. Secciani F, Bianchi L, Ermini L, Cianti R, Armini A, La Sala GB, et al. Protein profile of capacitated versus ejaculated human sperm. J Proteome Res. 2009;8(7):3377-89.

19. Bohring $C$, Krause $W$. The characterization of human spermatozoa membrane proteins-surface antigens and immunological infertility. Electrophoresis. 1999;20(4-5):971-6.

20. Bohring C, Krause W. Immune infertility: towards a better understanding of sperm (auto)-immunity. The value of proteomic analysis. Hum Reprod. 2003; 18(5):915-24.

21. Anderson NL, Anderson NG. Proteome and proteomics: new technologies, new concepts, and new words. Electrophoresis. 1998;19(11):1853-61.

22. Wu TF, Chu DS. Sperm chromatin: fertile grounds for proteomic discovery of clinical tools. Mol Cell Proteomics. 2008;7(10):1876-86.

23. Chan CC, Shui HA, Wu CH, Wang CY, Sun GH, Chen HM, et al. Motility and protein phosphorylation in healthy and asthenozoospermic sperm. J Proteome Res. 2009;8(11):5382-6.

24. Hashemitabar M, Sabbagh S, Orazizadeh M, Ghadiri A, Bahmanzadeh M. A proteomic analysis on human sperm tail: comparison between normozoospermia and asthenozoospermia. J Assist Reprod Genet. 2015; 32(6):853-63.

25. Miki K, Qu W, Goulding EH, Willis WD, Bunch DO, Strader LF, et al. Glyceraldehyde 3-phosphate dehydrogenase-S, a sperm-specific glycolytic enzyme, is required for sperm motility and male fertility. Proc Natl Acad Sci U S A. 2004;101(47):16501-6.

26. Ursini F, Heim S, Kiess M, Maiorino M, Roveri A, Wissing J, et al. Dual function of the selenoprotein PHGPx during sperm maturation. Science. 1999;285(5432):1393-6.

27. Pavlov E, Grigoriev SM, Dejean LM, Zweihorn CL, Mannella CA, Kinnally KW. The mitochondrial channel VDAC has a cation-selective open state. Biochim Biophys Acta. 2005;1710(2-3):96-102.

28. Choudhary OP, Ujwal R, Kowallis W, Coalson R, Abramson J, Grabe M. The electrostatics of VDAC: implications for selectivity and gating. J Mol Biol. 2010;396(3):580-92.

29. Kwon WS, Park YJ, Mohamed el SA, Pang MG. Voltage-dependent anion channels are a key factor of male fertility. Fertil Steril. 2013;99(2):354-61.

30. Turner RM. Moving to the beat: a review of mammalian sperm motility regulation. Reprod Fertil Dev. 2006;18(1-2):25-38.
31. Eddy EM, Toshimori K, O'Brien DA. Fibrous sheath of mammalian spermatozoa. Microsc Res Tech. 2003;61(1):103-15.

32. Miki K, Willis WD, Brown PR, Goulding EH, Fulcher KD, Eddy EM. Targeted disruption of the Akap4 gene causes defects in sperm flagellum and motility. Dev Biol. 2002;248(2):331-42.

33. Moretti E, Scapigliati G, Pascarelli NA, Baccetti B, Collodel G. Localization of AKAP4 and tubulin proteins in sperm with reduced motility. Asian J Androl. 2007;9(5):641-9.

34. Cao W, Gerton GL, Moss SB. Proteomic profiling of accessory structures from the mouse sperm flagellum. Mol Cell Proteomics. 2006;5(5):801-10.

35. Brohmann H, Pinnecke S, Hoyer-Fender S. Identification and characterization of new cDNAs encoding outer dense fiber proteins of rat sperm. J Biol Chem. 1997;272(15):10327-32.

36. Chemes HE, Olmedo SB, Carrere C, Oses R, Carizza C, Leisner M, et al. Ultrastructural pathology of the sperm flagellum: association between flagellar pathology and fertility prognosis in severely asthenozoospermic men. Hum Reprod. 1998;13(9):2521-6.

37. Petersen C, Fuzesi L, Hoyer-Fender S. Outer dense fibre proteins from human sperm tail: molecular cloning and expression analyses of two cDNA transcripts encoding proteins of approximately $70 \mathrm{kDa}$. Mol Hum Reprod. 1999:5(7):627-35.

38. Fujinoki M, Kawamura T, Toda $T$, Ohtake $H$, Shimizu N, Yamaoka S, et al. Identification of the 58-kDa phosphoprotein associated with motility initiation of hamster spermatozoa. J Biochem. 2003;134(4): 559-65.

39. Cosson J, White D, Huitorel P, Edde B, Cibert C, Audebert S, et al. Inhibition of flagellar beat frequency by a new anti-beta-tubulin antibody. Cell Motil Cytoskeleton. 1996;35(2):100-12.

40. Fackenthal JD, Turner FR, Raff EC. Tissue-specific microtubule functions in drosophila spermatogenesis require the beta 2-tubulin isotype-specific carboxy terminus. Dev Biol. 1993;158(1):213-27.

41. Neuer A, Spandorfer SD, Giraldo P, Dieterle S, Rosenwaks Z, Witkin SS. The role of heat shock proteins in reproduction. Hum Reprod Update. 2000;6(2): 149-59.

42. Schneider HC, Berthold J, Bauer MF, Dietmeier K, Guiard B, Brunner M, et al. Mitochondrial Hsp70/MIM44 complex facilitates protein import. Nature. 1994;371(6500):768-74

43. Aitken J, Krausz C, Buckingham D. Relationships between biochemical markers for residual sperm cytoplasm, reactive oxygen species generation, and the presence of leukocytes and precursor germ cells in human sperm suspensions. Mol Reprod Dev. 1994;39(3):268-79.

44. Siva AB, Kameshwari DB, Singh V, Pavani K, Sundaram CS, Rangaraj N, et al. Proteomics-based study on asthenozoospermia: differential expression of proteasome alpha complex. Mol Hum Reprod. 2010;16(7): 452-62.

45. Ergur AR, Dokras A, Giraldo JL, Habana A, Kovanci E, Huszar G. Sperm maturity and treatment choice of in vitro fertilization (IVF) or intracytoplasmic sperm injection: diminished sperm HspA2 chaperone levels predict IVF failure. Fertil Steril. 2002;77(5):910-8.

46. Cedenho AP, Lima SB, Cenedeze MA, Spaine DM, Ortiz V, Oehninger S. Oligozoospermia and heat-shock protein expression in ejaculated spermatozoa. Hum Reprod. 2006;21(7):1791-4.

47. Pizarro E, Pasten C, Kong M, Morales P. Proteasomal activity in mammalian spermatozoa. Mol Reprod Dev. 2004;69(1):87-93.

48. Sutovsky P, Hauser R, Sutovsky M. Increased levels of sperm ubiquitin correlate with semen quality in men from an andrology laboratory clinic population. Hum Reprod. 2004;19(3):628-38.

49. Sutovsky P, Turner RM, Hameed S, Sutovsky M. Differential ubiquitination of stallion sperm proteins: possible implications for infertility and reproductive seasonality. Biol Reprod. 2003;68(2):688-98.

50. Martinez-Heredia J, de Mateo S, Vidal-Taboada JM, Ballesca JL, Oliva R. Identification of proteomic differences in asthenozoospermic sperm samples. Hum Reprod. 2008;23(4):783-91.

51. Cardullo RA, Baltz JM. Metabolic regulation in mammalian sperm: mitochondrial volume determines sperm length and flagellar beat frequency. Cell Motil Cytoskeleton. 1991;19(3):180-8.

52. Coughlin EM, Christensen E, Kunz PL, Krishnamoorthy KS, Walker V, Dennis $\mathrm{NR}$, et al. Molecular analysis and prenatal diagnosis of human fumarase deficiency. Mol Genet Metab. 1998;63(4):254-62.

53. Caputo E, Manco G, Mandrich L, Guardiola J. A novel aspartyl proteinase from apocrine epithelia and breast tumors. J Biol Chem. 2000;275(11):7935-41. 
54. Lilja $\mathrm{H}$, Abrahamsson PA, Lundwall A. Semenogelin, the predominant protein in human semen. Primary structure and identification of closely related proteins in the male accessory sex glands and on the spermatozoa. J Biol Chem. 1989:264(3):1894-900.

55. Autiero M, Abrescia P, Guardiola J. Interaction of seminal plasma proteins with cell surface antigens: presence of a CD4-binding glycoprotein in human seminal plasma. Exp Cell Res. 1991;197(2):268-71.

56. Shen S, Wang J, Liang J, He D. Comparative proteomic study between human normal motility sperm and idiopathic asthenozoospermia. World J Urol. 2013;31 (6):1395-401.

57. Lachance C, Bailey JL, Leclerc P. Expression of Hsp60 and Grp78 in the human endometrium and oviduct, and their effect on sperm functions. Hum Reprod. 2007;22(10):2606-14.

58. Marin-Briggiler Cl, Gonzalez-Echeverria MF, Munuce MJ, Ghersevich S, Caille AM, Hellman U, et al. Glucose-regulated protein 78 (Grp78/BiP) is secreted by human oviduct epithelial cells and the recombinant protein modulates sperm-zona pellucida binding. Fertil Steril. 2010;93(5):1574-84.

59. Parte PP, Rao P, Redij S, Lobo V, D'Souza SJ, Gajbhiye R, et al. Sperm phosphoproteome profiling by ultra performance liquid chromatography followed by data independent analysis (LC-MS(E)) reveals altered proteomic signatures in asthenozoospermia. J Proteome. 2012;75(18):5861-71.

60. Wang J, Wang J, Zhang HR, Shi HJ, Ma D, Zhao HX, et al. Proteomic analysis of seminal plasma from asthenozoospermia patients reveals proteins that affect oxidative stress responses and semen quality. Asian J Androl. 2009; 11(4):484-91.

61. Saraswat M, Joenvaara S, Jain T, Tomar AK, Sinha A, Singh S, et al. Human spermatozoa quantitative proteomic signature classifies Normo- and Asthenozoospermia. Mol Cell Proteomics. 2017:16(1):57-72.

\section{Submit your next manuscript to BioMed Central and we will help you at every step:}

- We accept pre-submission inquiries

- Our selector tool helps you to find the most relevant journal

- We provide round the clock customer support

- Convenient online submission

- Thorough peer review

- Inclusion in PubMed and all major indexing services

- Maximum visibility for your research

Submit your manuscript at www.biomedcentral.com/submit

) Biomed Central 